\title{
Spectrally-Efficient 17.6-Tb/s DWDM Optical Transmission System over 678 km with Pre-Filtering Analysis
}

\author{
L. H. H. Carvalho, E. P. Silva, R. Silva, J. P. K Perin, J. C. R. F. Oliveira, \\ M. L. Silva, P. P. G. Cardoso and A. Paradisi \\ CPqD - Centro de Pesquisa e Desenvolvimento em Telecomunicações, Rod. Campinas/Mogi - Mirim, km \\ 118.5,SP - Campinas, Brazil, julioc@cpqd.com.br
}

\begin{abstract}
We present and analyze a 17.6-Tb/s DWDM optical transmission composed of 79 channels with 224-Gb/s RZ-DP16QAM modulation format per-channel and coherent detection. A maximum reach of $678 \mathrm{~km}$ is obtained with an optical recirculation loop composed by $226 \mathrm{~km}$ of pure-silica core fibers and EDFA amplification only in a 50-GHz grid system with spectral efficiency of 4.2-b/s/Hz. Also, a 25-GHz Gaussian optical pre-filter is applied to each channel and the same reach of $678 \mathrm{~km}$ is obtained in a 37.5 GHz channel grid system with spectral efficiency of $5.6-\mathrm{b} / \mathrm{s} / \mathrm{Hz}$. These results demonstrate a $33 \%$ system capacity improvement without transmission penalties.
\end{abstract}

Index Terms - Coherent detection, high-speed optical systems, modulation format, optical filtering, optical networks, spectral efficiency.

\section{INTRODUCTION}

Enabled by the increasing traffic in communications networks, which is mainly composed by highbandwidth data traffic, the optical fiber infrastructure is undergoing an evolution process in order to meet this wide variety of applications [1]. Optical networks have rapidly evolved in a way that, nowadays, presents reconfigurability characteristics and are capable of handling dense wavelengthdivision-multiplexing (DWDM) traffic with 100-Gb/s per-channel [2]. Today, high-speed systems targeting $400-\mathrm{Gb} / \mathrm{s}$ and 1-Tb/s per-channel are being intensively researched [3]. Since the beginning of its existence the optical transmission systems were based on intensity modulation (OOK) up to 10$\mathrm{Gb} / \mathrm{s}$. However, the increase of transmission rates and the spectral occupancy required for the maintenance of 50-GHz DWDM grid forced changes in the structure of transmission and reception in order to increase the spectral efficiency (SE) for systems with bit rates higher than 10-Gb/s. Transmission structures with phase modulation and differential coding (DQPSK) and interferometerbased reception for phase difference extraction were used for $40-\mathrm{Gb} / \mathrm{s}$ systems [4]. These systems improved the spectral efficiency from $0.5-\mathrm{b} / \mathrm{s} / \mathrm{Hz}(\mathrm{OOK})$ to $1-\mathrm{b} / \mathrm{s} / \mathrm{Hz}$ (DQPSK). A 2-b/s/Hz spectral efficiency is needed for 100-Gb/s channels to fit in the current 50-GHz DWDM grid. To reach such SE, polarization multiplexing together with the phase modulation was used to double the spectral efficiency. The use of dual-polarization quadrature phase-shift-keying (DP-QPSK) modulation format improved the SE to $2-\mathrm{b} / \mathrm{s} / \mathrm{Hz}$ and enabled the $100-\mathrm{Gb} / \mathrm{s}$ DWDM transmission in a $50-\mathrm{GHz}$ grid. However, coherent detection was needed in order to meet the phase recovery requirements. The use of 
coherent detection also enabled the total electrical field recovery, allowing the use of high-speed analog-to-digital converters (ADCs) for signal acquisition and digital signal processing (DSP) for digital coherency. The DSP is also used for chromatic dispersion compensation and polarization demultiplexing [5].

In order to meet the growing demand for transmission rates while maintaining the $100-\mathrm{Gb} / \mathrm{s}$ standardized [6] transmission and reception structures, the use of dual-polarization 16-quadrature amplitude modulation (DP-16QAM) modulation format is presented as a potential solution and its use in optical coherent systems is being intensively investigated [7]-[9]. The use of amplitude and phase modulation together with polarization multiplexing improves the SE from 2-b/s/Hz (DP-QPSK) to 4$\mathrm{b} / \mathrm{s} / \mathrm{Hz}$ (DP-16QAM), achieving a 200-Gb/s line rate while maintaining the same symbol rate. Basically the same 100G DP-QPSK transmission and reception structures are used for 200G DP16QAM systems, with the exception of the DSP algorithms for polarization demultiplexing and phase recovery. Another approach for spectral efficiency improvement is to make use of optical prefiltering, in which the modulated signal is filtered before being transmitted in order to reduce the signal bandwidth while maintaining the bit rate.

We demonstrate a 17.6-Tb/s DWDM optical system composed of 79 channels in a 50-GHz grid using 224-Gb/s RZ-DP-16QAM modulation format per-channel with 4.2-b/s/Hz SE and coherent detection with offline DSP. This result is the higher aggregate system bit rate reported in Latin America. A maximum reach of $678 \mathrm{~km}$ is obtained using an optical recirculation loop composed of $226 \mathrm{~km}$ of pure-silica core fiber (PSCF), 6 erbium-doped-fiber amplifiers (EDFAs) and a reconfigurable optical add/drop multiplexer (ROADM) based on flexible-grid wavelength selective switch (WSS) technology. We also perform optical pre-filtering in order to increase the system spectral efficiency. The same reach of $678 \mathrm{~km}$ is obtained when applying 25-GHz Gaussian prefiltering in each carrier before transmission and transmitting the channels in a $37.5-\mathrm{GHz}$ channel grid system, resulting in a spectral efficiency improvement from $4.2-\mathrm{b} / \mathrm{s} / \mathrm{Hz}$ to $5.6-\mathrm{b} / \mathrm{s} / \mathrm{Hz}$ without transmission penalties. The different channel grid is enabled by the use of a flexible-grid wavelength selective switch (WSS) with 12.5-GHz granularity.

\section{EXPERIMENTAL SETUP}

The experimental setup is depicted in Fig. 1. and it can be divided into three main subsystems: transmission, recirculation loop and reception, as illustrated in Fig. 1.a, 1.b and 1.c, respectively. The transmission subsystem consisted of two sets of optical sources (carriers), as depicted in Fig. 1.a. One is defined by 78 distributed-feedback (DFB) lasers of 2-MHz linewidth in the 50-GHz DWDM grid. The other one refers to the channel under test, in which was used an external-cavity laser (ECL) of $100-\mathrm{kHz}$ linewidth. Each set of carriers was sent to an independet DP-QPSK optical modulator, in order to generate 79 channels with 112-Gb/s DP-QPSK modulation per-channel, which is $100-\mathrm{Gb} / \mathrm{s}$ of line rate and 12-Gb/s of FEC, Ethernet protocols and headers. Four streams of uncorrelated pseudo- 
random bit sequences (PRBS) with $2^{15}-1$ length at $28-\mathrm{GHz}$ were used to modulate each carrier into a 112-Gb/s PDM-QPSK signal. The 112-Gb/s signals were sent to a pulse carver for RZ pulse shaping, which also acted as a linear polarizer. With a specific state-of-polarization (SOP) input, the 56-Gb/s QPSK signals in each polarization of each carrier were combined into a single-polarization signal inside the pulse carver, and a single-polarization 112-Gb/s RZ-16-QAM was obtained in each carrier at the output, as described in [10]. A polarization multiplexing stage (PDM emulator) was used to generate a 224-Gb/s RZ-PDM-16-QAM signal in each carrier. The optical eye-diagrams for the 112$\mathrm{Gb} / \mathrm{s}$ RZ-16QAM and for the 224-Gb/s RZ-DP-16QAM are depicted in Fig. 1. All the carriers were combined by a waveshaper in order to generate a 79-channel DWDM system with 17.6-Tb/s aggregate bit rate and 224-Gb/s per-channel, as depicted in Fig. 1. The waveshaper was also used to apply the optical pre-filtering in each carrier before transmission.

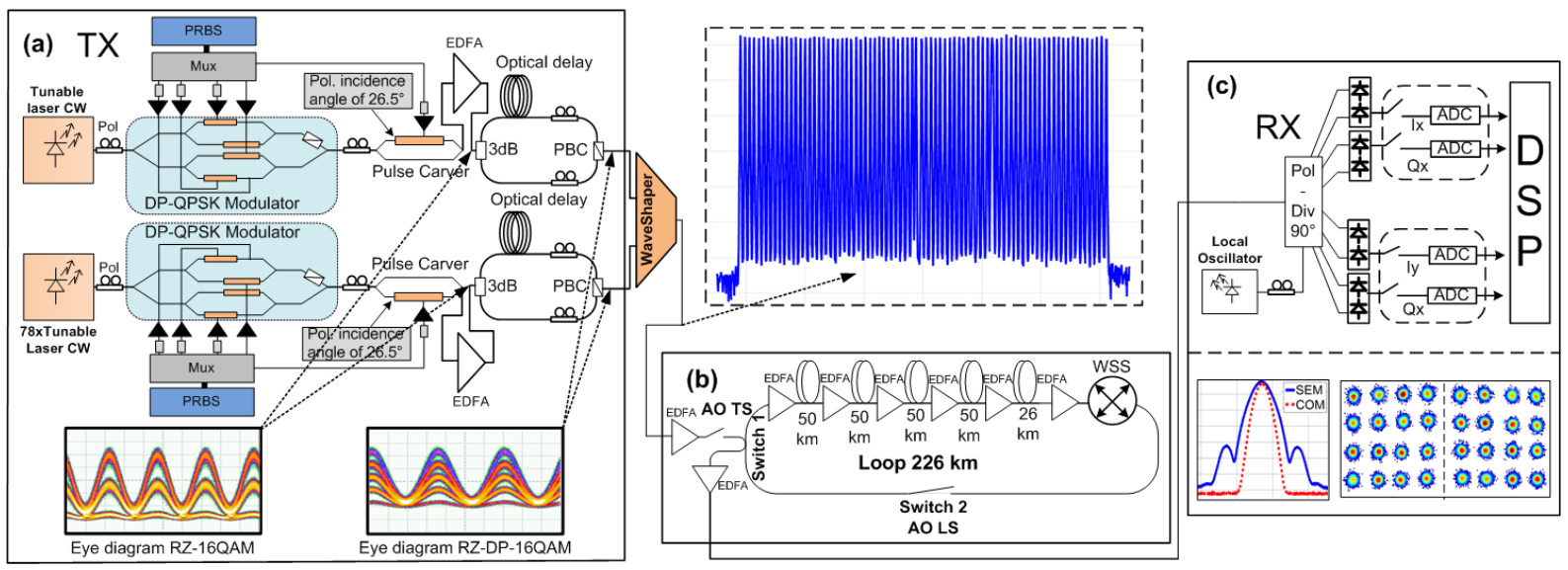

Fig. 1. Experimental setup. (a) Transmission; (b) recirculation loop; (c) reception. The transmitted DWDM spectrum, as well as the tested channel spectrum with and without pre-filtering and the received constellation are also illustrated.

The optical recirculation loop structure is detailed in Fig. 1b. An EDFA is used at the loop input in order to compensate losses from passive components and to reach the required input power for the loop operation. The operation of the recirculation system is controlled by two acousto-optic switches (AOS) operating in a complementary mode, which means that at the instant when one switch is open the other one is closed. This operation enables the signal to propagate through the system by any desired number of loop rounds in order to evaluate the maximum transmission reach. The total fiber length is composed of four spans of $50 \mathrm{~km}$ and one span of $26 \mathrm{~km}$, all spans of PSCF with 0.176 $\mathrm{dB} / \mathrm{km}$ of attenuation, $80 \mu \mathrm{m} 2$ of effective area, $18.6 \mathrm{ps} / \mathrm{nm}$ of chromatic dispersion (CD) and 0.0665 $\mathrm{ps} / \sqrt{\mathrm{km}}$ of polarization mode dispersion (PMD) at $1550 \mathrm{~nm}$. The $50 \mathrm{~km}$ span losses of $\sim 9 \mathrm{~dB}$ are compensated by EDFAs with co-propagating $980 \mathrm{~nm}$ pumps, operating in the power control mode with $17 \mathrm{dBm}$ output power. Three gain flattening filters (GFFs) are also used inside the loop in order to reduce the flatness penalties caused by the cascaded EDFAs. A flexible-grid WSS is used to equalize the DWDM spectrum after each loop round and to impose the channel grid (50-GHz or 37.5GHz). The experimented system scenarios are depicted in Fig. 2. Fig. 2.a depicts a 50-GHz spacing DWDM system with 79 channels, 224-Gb/s RZ-DP-16QAM per channel and 50-GHz ROADM 
filtering. Fig 2.b depicts a 37.5-GHz channel grid DWDM optical system composed of 79 channels with 224-Gb/s RZ-DP-16QAM modulation format and 25-GHz Gaussian optical pre-filtering per channel. In order to validate the transmission of RZ-DP-16QAM in a constrained bandwidth system, the optical channel filtering imposed by the flexible-grid WSS is modified from standard $50-\mathrm{GHz}$ (Fig. 2.a) to a 37.5-GHz narrow-bandwidth filtering (Fig. 2.b). This can be achieved due to the flexible-grid technology, which allows the user to define custom channel filtering with $12.5-\mathrm{GHz}$ granularity.
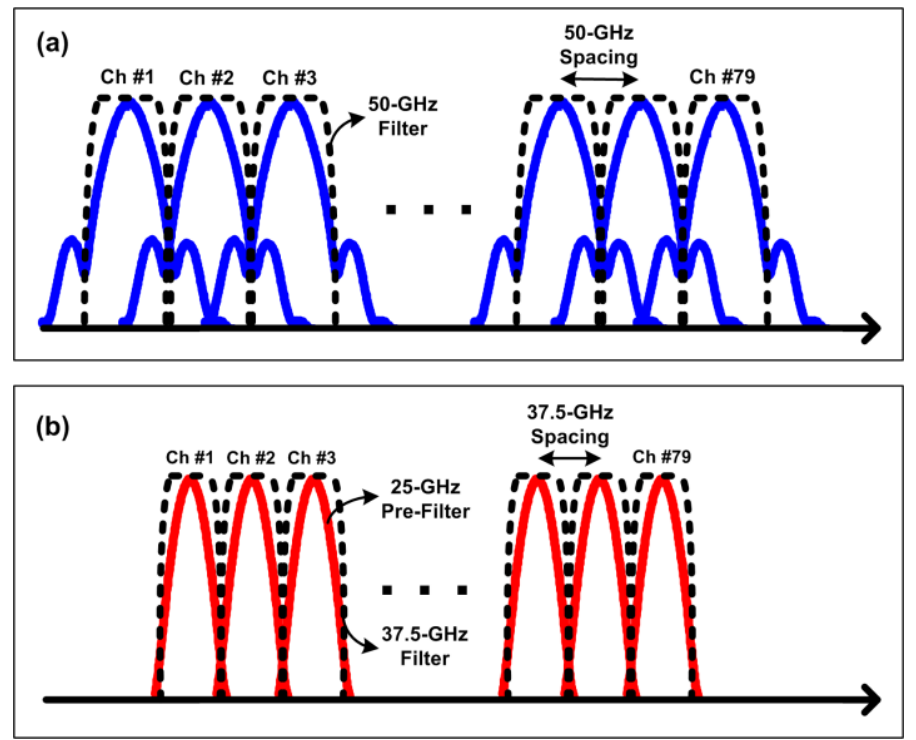

Fig. 2. DWDM system scenarios with 79x224-Gb/s RZ-DP-16QAM channels. (a) 50-GHz channel grid;

(b) 25-GHz optical pre-filtering and 37.5-GHz channel grid.

A synchronous triggering system allows the AOS to switch the signal and the receiver to acquire the signal after a specified number of round loops, allowing the system evaluation in a long-haul scenario. The recirculation loop output is amplified before the reception stage. Fig 1.c depicts the block diagram of the coherent reception structure used to recover the transmitted signal. The reception structure can be divided into three principal components: opto-electric (O-E) conversion, analog-todigital conversion (ADC) and digital signal processing (DSP). The opto-electric conversion is realized by a set of devices: local oscillator (LO), $2 \times 890^{\circ}$ hybrid and balanced photodiodes. This scheme is responsible to convert the modulated optical signal into a baseband electrical signal with separated IQ and polarization components. The $20-\mathrm{GHz}$ ADC acquires the baseband electrical signal with a $40-$ GS/s sampling rate and the digitalized data is stored for offline processing. A set of DSP algorithms are used to compensate the degrading effects imposed by the channel (chromatic dispersion, polarization-dependent losses, devices imperfection, etc.) as well as to realize the coherency in the digital domain by the use of frequency and phase recovery algorithms. After the DSP we obtain the constellation diagram as well as the estimated and counted bit-error-rates (BER) in order to evaluate the system performance. The optical spectrum and constellation diagrams for the 224-Gb/s RZ-DP16QAM signals with and without optical pre-filtering are also depicted in Fig. 1. 


\section{DIGITAL SIGNAL PROCESSING}

The use of coherent detection, which implies the use of a local oscillator and an analog-to-digital converter, enables the application of digital signal processing techniques on the acquired signal in order to minimize the physical-layer effects imposed by the communications channel and to completely recover the frequency and phase of the signal.

The algorithms sequence used in the DSP unit is depicted in Fig. 3. A deskew algorithm is used to compensate for the electrical paths differences (different electrical cables), while the orthonormalization compensates the I/Q components distortions and the $90^{\circ}$ Hybrid imperfections using the Gram-Schmidt algorithm [11]. The total chromatic dispersion imposed by the transmission link is fully compensated using the time-domain CD equalizer, as in [12]. The coefficients and length of a finite impulse response (FIR) filter are calculated and used to equalize the signal by applying the CD inverse transfer function.

A timing recovery based on Gardner algorithm [13] recovers the correct symbol time. The dynamic equalizer separates the orthogonal polarizations and also compensates the residual CD and the PMD. A 2x2 multiple-input multiple-output (MIMO) constant modulus algorithm (CMA) is used for the dynamic equalizer, using four FIR filters [5], [11]-[13], as depicted in Fig. 3.

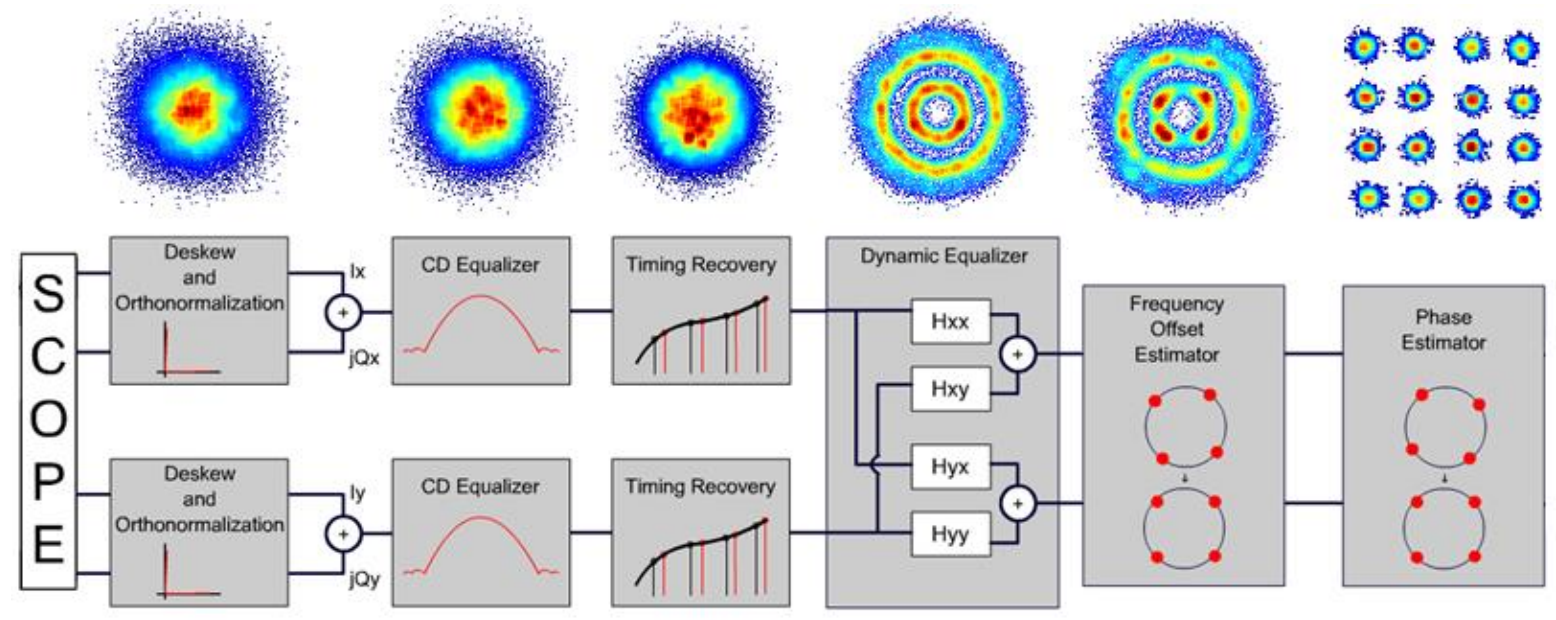

Fig. 3. Functional diagram of the DSP unit.

The last step is to implement the digital coherency (or carrier recovery), by the use of frequency and phase recovery algorithms. The frequency estimation is implemented as in [14], while a feed-forward phase estimation (FFPE) is used for phase recovery [15]. A constellation diagram, as illustrated in Fig. 3., is the output for the set of DSP algorithms, which can be used for symbol decision, BER counting and BER estimation. Fig. 3. also illustrate the signal constellation evolution after each DSP block. The received signal is penalized by the physical-layer impairments, which the chromatic dispersion and PMD are the principal contributors for the bit spreading. However, these effects are fully compensated by the digital filtering. 


\section{RESULTS}

This section presents the obtained experimental results. The measured back-to-back curves for the 224-Gb/s RZ-DP-16QAM, 224-Gb/s RZ-DP-16QAM with 25-GHz Gaussian filtering and 224-Gb/s NRZ-DP-16QAM with 25-GHz Gaussian filtering channels, as well as the theoretical back-to-back curve for 224-Gb/s DP-16QAM are depicted in Fig. 4. For the 224-Gb/s RZ-DP-16QAM, the required OSNR/0.1 nm for a BER of $1 \times 10^{-3}$ is $23.5 \mathrm{~dB}$, which is a $\sim 3.5 \mathrm{~dB}$ penalty with respect to theory. An additional $\sim 0.1 \mathrm{~dB}$ penalty is obtained from filtering the $224-\mathrm{Gb} / \mathrm{s} \mathrm{RZ}$ signal, which can be considered a negligible filtering penalty. However, an additional $\sim 2.5 \mathrm{~dB}$ penalty is obtained from filtering the 224-Gb/s NRZ signal. This verifies the filtering resilience of the RZ channel, which is enabled by the spectrum shape of the RZ pulse that acts as a signal pre-emphasis to reduce the intersymbol interference (ISI) caused by the filtering, resulting in an increase of spectral efficiency with negligible penalties. The required OSNR/0.1 nm at the FEC limit of $3.8 \times 10^{-3}$ for the 224-Gb/s RZ-DP-16QAM is $\sim 19.5 \mathrm{~dB}$ and for the 224-Gb/s RZ-DP-16QAM with $25-\mathrm{GHz}$ Gaussian filtering is $\sim 20 \mathrm{~dB}$.

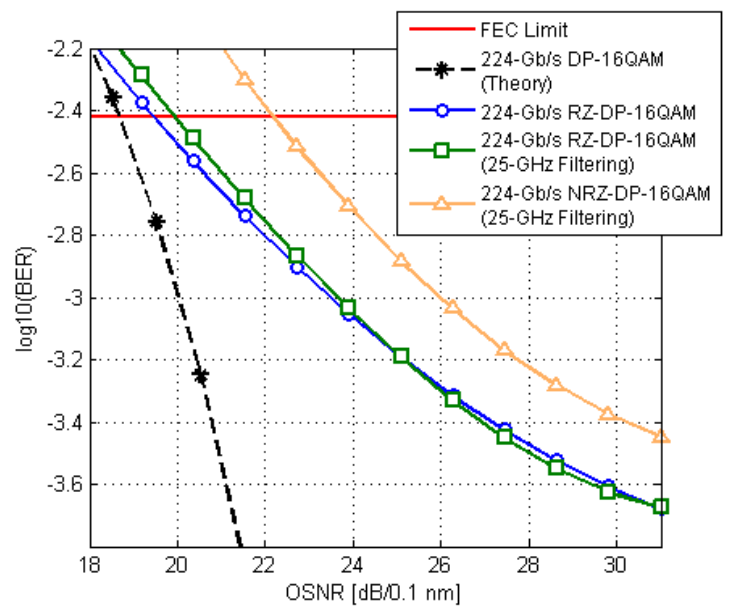

Fig. 4. Back-to-back curves, measured and theoretical.

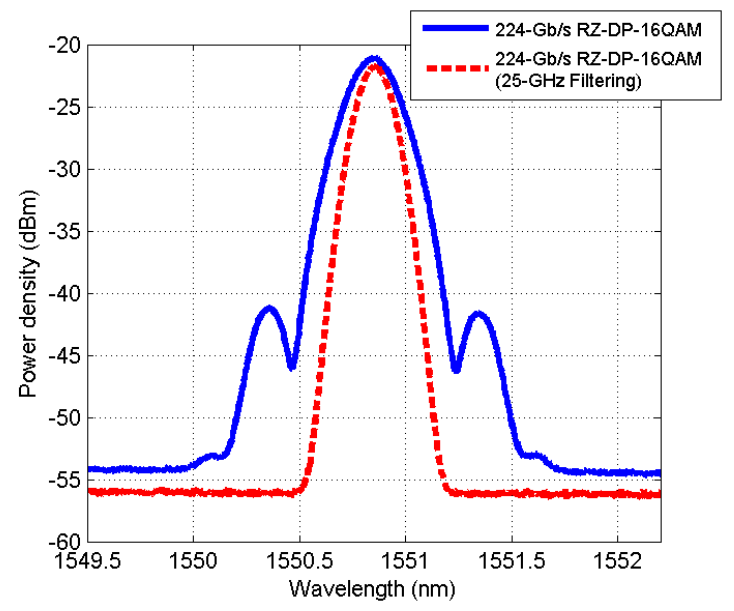

Fig. 5. Spectrum of a 224-Gb/s RZ-DP-16QAM signal with and without $25-\mathrm{GHz}$ filtering.

The optical spectrums of the 224-Gb/s RZ-DP-16QAM with and without 25-GHz optical filtering are depicted in Fig. 5. The spectral-component losses and the bandwidth narrowing due to the filtering can be observed. However, as verified in Fig. 4., the OSNR penalty due to the filtering in the RZ signal is considered negligible. The RZ pulse shaping with optical pre-filtering will be used in order to increase the system spectral efficiency.

The transmission results in terms of BER in function of the reach for the $50-\mathrm{GHz}$ and $37.5-\mathrm{GHz}$ channel grid systems are depicted in Fig. 6. The 50-GHz channel grid system is composed of 79 channels with 224-Gb/s RZ-DP-16QAM modulation format and 4.2-b/s/Hz SE, while the 37.5-GHz channel grid system is a composed of 79 channels with 224-Gb/s RZ-DP-16QAM pre-filtered with 25-GHz Gaussian optical filters and 5.6-b/s/Hz SE. The total power inserted in the optical fiber is $\sim 17$ $\mathrm{dBm}$ for both systems, which implies in a -2 $\mathrm{dBm}$ launch power per-channel. A maximum reach of $678 \mathrm{~km}$ is obtained for both systems, which is the equivalent of three 226-km loop rounds. The 
received OSNR for the 50 and $37.5-\mathrm{GHz}$ channel grid systems are $23.6 \mathrm{~dB}$ and $23.8 \mathrm{~dB}$, which results in a total transmission penalty of $4.1 \mathrm{~dB}$ and $3.8 \mathrm{~dB}$, respectively.

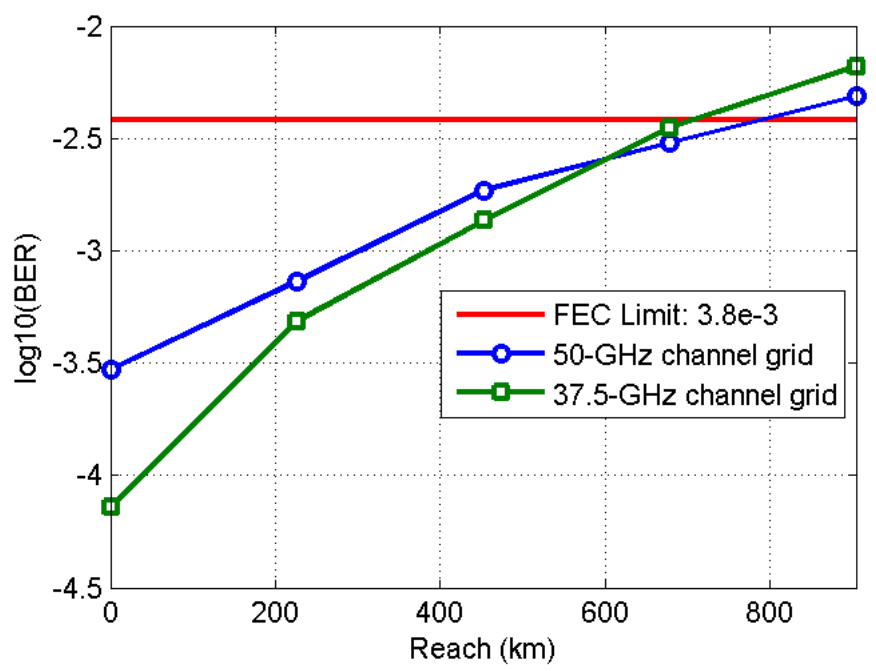

Fig. 6. Transmission reach results.

Although the systems present the same maxmim reach, the use of optical pre-filtering increases the system spectral efficiency. A system with 224-Gb/s DP-16QAM at 28-GBd in a 50-GHz channel grid presents a spectral efficiency of $4.2-\mathrm{b} / \mathrm{s} / \mathrm{Hz}$, while the same system with $25-\mathrm{GHz}$ optical pre-filtering in a $37.5-\mathrm{GHz}$ channel grid scenario presents a spectral efficiency of $5.6-\mathrm{b} / \mathrm{s} / \mathrm{Hz}$, which represents an increase of 33\% in the system capacity. The use of higher-order QAM signals is another approach to increase the system SE. However, the requirements in terms of laser phase noise, received OSNR, nonlinear threshold and $\mathrm{ADC}$ effective number of bits $(\mathrm{ENoB})$ are more stringent for these signals, which turns the use of 16QAM modulation a suitable approach for next generation of optical transmission. Although channel grids lower than $50-\mathrm{GHz}$ are not commercially available, the use of flexible-grid WSSs enables the construction ROADMs with variable bandwidth in a $12.5-\mathrm{GHz}$ granularity. The use of optical pre-filtering in conjunction with flexible-grid ROADMs composes a simple and efficient solution to increase the capacity of the current optical systems.

\section{CONCLUSION}

We demonstrate a spectrally-efficient 17-Tb/s (79×224-Gb/s RZ-DP-16QAM) DWDM optical transmission system with coherent detection and offline DSP. Using the current 50-GHz DWDM grid, a maximum reach of $678 \mathrm{~km}$ is obtained with a $4.2-\mathrm{b} / \mathrm{s} / \mathrm{Hz}$ spectral efficiency. The use of RZ pulse shaping with optical pre-filtering allowed the transmission in a $37.5-\mathrm{GHz}$ channel grid system with a $5.6-\mathrm{b} / \mathrm{s} / \mathrm{Hz}$ SE in the same reach of $678 \mathrm{~km}$, which results in a $33 \%$ improvement in the system capacity without transmission penalties. These results were obtained in a scenario with EDFA amplification only, showing the robustness of the proposed system in a real network scenario.

The optical pre-filtering increases the system spectral efficiency, allowing the allocation of a higher number of channels in the same bandwidth and maintaining the modulation format at a penalty cost of 
transmission performance due to the intersymbol interference caused by the filtering. Thus, prefiltering systems should be designed considering a trade-off between spectral efficiency, performance and maximum reach. This is an important factor when we consider that the future of optical networks tends to flexible applications of bit rate, modulation formats and channel bandwidth.

\section{ACKNOWLEDGMENT}

This work has been sponsored by Funttel/Finep: GIGA Project, phase 2, and PAR100GETH.

\section{REFERENCES}

[1] Renaudier, J., Bertran-Pardo, O., Charlet, G., Salsi, M., Mardoyan, H., Tran, P. eBigo, S., "8 Tb/s long haul transmission over low dispersion fibers using $100 \mathrm{~Gb} / \mathrm{s}$ PDM-QPSK," Bell Labs Technical Journal 14(4), 27-46 (2010).

[2] A. Nag, M. Tornatore, e B. Mukherjee, "Optical Network Design with Mixed Line Rates and Multiple Modulation Formats", IEEE/OSA J. of Lightwave Technology, V. 28, N. 4, pp.466 - 475, 2010.

[3] S. Chandrasekhar e Xiang Liu, "Terabit Superchannels for High Spectral Efficiency Transmission", ECOC 2010, Tu.3.C.5, Sep. 2010.

[4] Chandrasekhar, S. and Liu, X., "40 Gb/s DBPSK e DQPSK formats for transparent $50 \mathrm{GHz}$ DWDM transmission,” Bell Labs Technical Journal 14(4), 11-26 (2010).

[5] S.J. Savory, "Digital Coherent Optical Receivers: Algorithms and Subsystems," Selected Topics in Quantum Electronics, IEEE Journal of, vol.16, no.5, pp.1164-1179, Sept.-Oct. 2010.

[6] Optical Internetworking Forum, "Implementation Agreement for Integrated Polarization Multiplexed Quadrature Modulated Transmitters", IA \# OIF-PMQ-TX-01.0, Mar. 2010.

[7] A. H. Gnauck, P. J. Winzer, S. Chandrasekhar, X. Liu, B. Zhu and D. W. Peckham, "Spectrally Efficient Long-Haul WDM Transmission Using 224-Gb/s Polarization-Multiplexed 16-QAM", journal of lightwave technology, vol. 29 , no. 4, pp. 373-377, February 15, 2011.

[8] P. J. Winzer, A. H. Gnauck, S. Chandrasekhar, S. Draving, J. Evangelista and B. Zhu, "Generation and 1,200-km Transmission of 448-Gb/s ETDM 56-Gbaud PDM 16-QAM using a Single I/Q Modulator", Optical Communication (ECOC), 2010 36th European Conference and Exhibition.

[9] Carsten Behrens, Sergejs Makovejs, Robert I. Killey, Seb. J. Savory, Ming Chen, and Polina Bayvel, "Pulse-shaping versus digital backpropagation in 224Gbit/s PDM-16QAM transmission.", Optics Express, Vol. 19, Issue 14, pp. 12879-12884, 2011.

[10] I. Morohashi, M. Sudo, T. Sakamoto, A. Kanno, A. Chiba, J. Ichikawa and T. Kawanishi, "16 QAM Synthesis by Angular Superposition of Polarization using Dual-Polarization QPSK Modulator”, ECOC 2010, P3.14, Sep. 2010.

[11] S. Haykin, Adaptive Filter Theory, 4th Ed., Prentice Hall, 2001.

[12] S. J. Savory, "Digital filters for coherent optical receivers," Opt. Express 16, 804-817 (2008).

[13] F. Gardner, “A BPSK/QPSK timing-error detector for sampled receivers," IEEE Trans. Commun., vol. COM-34, no. 5, pp. 423-429, May 1986.

[14] Z. Li et al., "Wide-Range and Fast-Convergence Frequency Offset Estimator by BER-Aiding...”OSA/ACP 2009, ThT2, (2009).

[15] El-Darawy, M.; Pfau, T.; Hoffmann, S.; Noe, R.;, "Differential phase compensated constant modulus algorithm for phase noise tolerant coherent optical transmission," Summer Topical Meeting, 2009. LEOSST '09.IEEE/LEOS, pp.9596, 20-22 July 2009. 